\title{
Experimental determination of the Rashba Coefficient in InSb/InAlSb quantum wells at zero magnetic field and elevated temperatures
}

\author{
M A Leontiadou ${ }^{1}, \mathrm{~K}$ L Litvinenko ${ }^{1}$, A M Gilbertson ${ }^{2}, \mathrm{C}$ R Pidgeon ${ }^{3}$, W R Branford ${ }^{2}$, \\ L F Cohen ${ }^{2}$, M Fearn ${ }^{4}$, T Ashley ${ }^{4}$, M T Emeny ${ }^{4}$, B N Murdin ${ }^{1}$ and S K Clowes ${ }^{1 *}$. \\ ${ }^{1}$ Advanced Technology Institute, University of Surrey, Guildford GU2 7XH, England \\ ${ }^{2}$ Blackett Laboratory, Imperial College, London, SW7 2BZ, England \\ ${ }^{3}$ Department of Physics, Heriot-Watt University, Edinburgh EH14 4AS, Scotland \\ ${ }^{4}$ QinetiQ Ltd., St Andrews Road, Malvern, WR14 3PS, England \\ *Email: s.clowes@surrey.ac.uk
}

\begin{abstract}
We report the optical measurement of the spin dynamics at elevated temperatures and in zero magnetic field, for two types of degenerately doped n-InSb quantum wells (QWs), one asymmetric (sample A) and one symmetric (sample B) with regards to the electrostatic potential across the QW. Making use of three directly determined experimental parameters: the spin lifetime, $\tau_{\mathrm{s}}$, the sheet carrier concentration, $n$, and the electron mobility, $\mu$, we directly extract the zero field spin splitting. For the asymmetric sample where the Rashba interaction is the dominant source of spin splitting, we deduce a room temperature Rashba parameter of $\alpha=$ $0.09 \pm 0.1 \mathrm{eVA}$ which is in good agreement with calculations and we estimate the Rashba coefficient $\alpha_{0}$ (a figure of merit for the ease with which electron spins can be modulated via an electric field). We review the merits/limitations of this approach and the implications of our finding for spintronic devices.
\end{abstract}

\section{Introduction}

The realisation of efficient semiconductor based spin filters and manipulators is essential for semiconductor spintronics to achieve its promised potential as a route to faster and more energy efficient electronics. One of the challenges is the creation of spin polarised currents within the inherently non-magnetic semiconductor. Conventional approaches to this involve the spin injection from ferromagnetic electrodes or diluting semiconductors with magnetic material. However it was demonstrated that spin polarised electric currents can be produced in semiconductors without the use of magnetic materials[1]. In principle, it may be possible to produce very high efficiency spin filters by spin sensitive reflection or refraction of ballistic electrons in two dimensional semiconductors[2-4]. A crucial ingredient for these approaches is a strong spin splitting in zero magnetic field, $\Delta E_{R}$, due to structural inversion asymmetry (SIA) in quantum wells (QWs). This is characterised by the Rashba parameter $\alpha$, where $\Delta E_{\mathrm{R}}=2 \alpha k$, with $k$ the electron momentum[5-7]. The Rashba interaction is important for semiconductor spintronics because its magnitude $(\alpha)$, and therefore $\Delta E_{\mathrm{R}}$ can be modulated via an applied electric field $F$. Accordingly, we emphasise that it is not simply the magnitude of $\Delta E_{\mathrm{R}}$ that is important, but also the ease with which an applied electric field, $F$, can modulate this effect. $\alpha$ is often related to $F$ through the linear expression $\alpha=e \alpha_{0} F$, where $\alpha_{0}$ is the socalled Rashba coefficient, related to the band structure of the host crystal[8], and is predicted to be largest in materials with narrow band gaps and high spin-orbit splitting such as $\mathrm{InSb}$ [9]. While this linear relationship is not strictly accurate[10], the conceptual $\alpha_{0}$ remains a valid figure of merit to compare the relative spintronic potential of different materials - the experimental knowledge of which is essential. 
Experimental determination of the Rashba Coefficient in InSb/InAlSb quantum wells at zero magnetic field and elevated temperatures

In practice, the Rashba spin splitting $\Delta E_{\mathrm{R}}$ is difficult to measure. The common techniques for measuring it require either extrapolation from high magnetic fields[11, 12] or the fitting of quantum interference corrections to the magnetoconductivity at low fields[13]. Extrapolation from high magnetic fields is non-trivial, not-only because the Zeeman effect obscures the Rashba contribution to the splitting, but also because the latter is rapidly quenched by the field[14]. Thus neither technique is a simple or direct measurement of spin splitting at zero field, the value relevant for most practical applications. Furthermore, the measurements listed above are restricted to low temperatures where the necessary quantum mechanical effects persist. In contrast, optical measurements of the spin relaxation in zero magnetic field provide an alternative measure of the spin splitting in the conduction bands[8]. For sufficiently large zero-field spin splitting (which covers most cases of interest) the spin dynamics are directly influenced by the size of the zero-field spin splitting (ZFSS), and so inversely, measurement of the spin dynamics directly reveals the spin splitting.

In the present work we report optical measurements of the spin dynamics at zero magnetic field and elevated temperatures $(77 \mathrm{~K}$ to $300 \mathrm{~K}$ ) for two degenerate n-InSb/InAlSb QWs: (A asymmetric and B - symmetric).

\section{THEORY}

In the absence of an applied magnetic field the conduction band of low-dimensional III-V semiconductor structures is spin-split by spin-orbit effects that arise from the lack of inversion symmetry, with contributions from both bulk inversion asymmetry (BIA) and structural inversion asymmetry (SIA). BIA results from the symmetry of the zinc blende lattice, which in a QW leads to the so-called Dresselhaus[15] spin-splitting terms, one linear and one cubic in the in-plane momentum. SIA arises from the asymmetry of the QW structure, leading to the Rashba spin splitting term which is linear in the momentum[5-8, 16].

For sufficiently large ZFSS, spin relaxation is dominated by the D'yakonov-Perel (DP) process[16-20]. The DP process arises from fact that this spin-splitting, in contrast with the well known Zeeman splitting, corresponds to effective magnetic fields that are momentum dependent. In this scenario, since each electron has different momentum its spin precesses about a different Larmor precession vector, $\boldsymbol{\Omega}(\mathbf{k})$. Any macroscopic polarization (produced by optical or electrical injection) thus quickly dephases - the rate is determined by the size of the spin splitting. This dephasing is slowed by scattering: if $\mathbf{k}$ performs a random walk then so does $\boldsymbol{\Omega}(\mathbf{k})$ so that the spin vectors dephase diffusively. In this case the spin relaxation rate is described by

$$
\frac{1}{\tau_{s}}=\left\langle\left|\boldsymbol{\Omega}_{\perp}\right|^{2}\right\rangle \tau_{p}^{*}
$$

where $\tau_{p}^{*}$ is the total momentum scattering time including the effects of both inelastic and elastic electron-electron scattering[16], $\boldsymbol{\Omega}_{\perp}$ is the component of $\boldsymbol{\Omega}(\mathbf{k})$ perpendicular to the spin $\boldsymbol{\sigma}$, and

$$
\boldsymbol{\Omega}(\mathbf{k})=\frac{1}{\hbar}\left(\begin{array}{c}
\beta k_{x}-\alpha k_{y}-\gamma k_{x} k_{y}^{2} \\
\alpha k_{x}-\beta k_{y}+\gamma k_{x}^{2} k_{y} \\
0
\end{array}\right)
$$

Here, $\alpha, \beta$ and $\gamma$ parameterize the strengths of the Rashba, k-linear Dresselhaus and k-cubic Dresselhaus spin splittings, respectively. Here, the corresponding ZFSS of the conduction band is $\Delta E_{R}(k)=2 \hbar|\Omega|$. Note that both $\beta$ and $\gamma$ are positive in III-V semiconductors. 
Experimental determination of the Rashba Coefficient in InSb/InAlSb quantum wells at zero magnetic field and elevated temperatures

The DP spin relaxation rate for electrons at the Fermi energy (appropriate for a degenerately doped quantum well) which have been oriented along the [001] direction, appropriate for optical excitation is[17, 20]:

$$
\frac{1}{\tau_{s}^{[001]}}=\frac{4 k_{F}^{2}}{\hbar^{2}}\left\{\alpha^{2}+\left(\beta-\frac{1}{4} \gamma k_{F}^{2}\right)^{2}+\frac{1}{16} \gamma^{2} k_{F}^{4} \frac{\tau_{3}}{\tau_{1}}\right\} \tau_{1}
$$

where $k_{F}=\sqrt{2 \pi n}$ is the Fermi wave-vector. The lifetimes $\tau_{1}$ and $\tau_{3}$ are reciprocals of the angular components of the momentum scattering rate, so that for isotropic scattering [21] (appropriate for a uniformly doped QW) $\tau_{1}=\tau_{3}$ while for small-angle scattering (modulation doped structures) $\tau_{3} / \tau_{1}$ is small[17, 22].

It is of practical interest to define, using Eq. (3), an effective inversion asymmetry spin splitting parameter, $\alpha^{*}$ (with dimensions of eVA) by,

$$
\alpha^{* 2}(T)=\frac{\hbar^{2}}{8 \pi n(T) \tau_{p}(T) \tau_{s}(T)}
$$

where $\tau_{\mathrm{p}}$ is the inelastic scattering, and $\Delta E^{*}=2 \alpha^{*} k_{F}$. $\alpha^{*}$ may be determined purely from experimentally measurable quantities $n, \tau_{\mathrm{p}}$ and $\tau_{\mathrm{s}}$. Substitution of Eq. (3) leads to

$$
\alpha^{*^{2}}(T)=\alpha^{2}+\left(\beta-\frac{1}{4} \gamma k_{F}^{2}\right)^{2}+\frac{1}{16} \gamma^{2} k_{F}^{4} \frac{\tau_{3}}{\tau_{p}}
$$

for the specific case of a degenerate $\mathrm{QW}$ in the absence of electron-electron scattering, so that $\tau_{1}=\tau_{\mathrm{p}}{ }^{*}$ $=\tau_{\mathrm{p}}$. We see that if the Rashba contribution dominates, Eq. (5) simplifies to $\alpha^{*} \approx \alpha$, and $\Delta E^{*}=\Delta E_{\mathrm{R}}$. It can be seen from Eq. (5) that $\alpha^{*}$ has no explicit temperature dependence, although as discussed in the following section and evidenced in Fig. 2 each parameter has an implicit temperature dependence. We now have a route to determine the Rashba parameter, albeit only for asymmetric QWs, using the optically measured spin dynamics without the constraints of low temperatures and high magnetic fields associated with alternative electrical techniques.

\section{EXPERIMENT}

We investigated two $20 \mathrm{~nm} n$-InSb/AlInSb single QW samples grown by molecular beam epitaxial onto semi insulating [001] GaAs substrates. In both samples the QW is confined on each side by an $\mathrm{In}_{0.85} \mathrm{Al}_{0.15} \mathrm{Sb}$ barrier. Sample A (me1833) was Te modulation doped $20 \mathrm{~nm}$ above the QW whilst sample B (me1831F) was uniformly Te doped in the QW region. Self-consistent Schrödinger-Poisson (SP) solutions for the band profile of samples A and B at $77 \mathrm{~K}$ are shown in Fig. 1(a) and (b), respectively. For the purposes of our experiment, these samples provide structures with very different electrostatic profiles and we expect the values of $\alpha^{*}$ to reflect this. The asymmetric doping scheme of sample A results in a large built-in electric field that is expected to enhance $\alpha$ (and hence $\alpha^{*}$ ) with respect to $\beta$ and $\gamma$ in Eqs. (3) and (5), and thus we expect $\alpha^{*}$ to be enhanced with respect to sample B. The sheet density and mobility were obtained for the temperature range $77 \mathrm{~K}$ to $300 \mathrm{~K}$, by standard low field Hall Effect measurements. Optical measurements of the spin dynamics were performed in zero magnetic field using the circularly polarised pump-probe absorption technique described in detail elsewhere[23]. The samples were pumped using $250 \mathrm{kHz} 1 \mathrm{~mW}$ laser pulses at $3.4 \mu \mathrm{m}$ wavelength. However, due to the required optical components and the narrow width of the QW only a small fraction $(\sim 0.025 \%)$ is actually absorbed. This corresponds to an excited electron density of $\sim 5 \times 10^{10}$ $\mathrm{cm}^{-2}$. This is significantly lower than the doped electron density and therefore has negligible effect on $k_{\mathrm{F}}$. 
Experimental determination of the Rashba Coefficient in InSb/InAlSb quantum wells at zero magnetic field and elevated temperatures

\section{RESULTS AND DISCUSSION}

Figures 2(a) shows the results for the temperature dependence of the sheet density $n$. The minimum room-temperature carrier concentration encountered (sample A) was $3.5 \times 10^{11} \mathrm{~cm}^{-2}$, corresponding to a Fermi energy $E_{F} \approx 40 \mathrm{meV}$ substantially greater than $k_{B} T=26 \mathrm{meV}$. All samples were therefore degenerate over the entire temperature range. We have assumed in our analysis that electron-electron scattering is negligible owing to the relatively low carrier density of our samples, and therefore that $\tau_{p}^{*} \approx \tau_{p}$. Note that if electron-electron scattering were to be important, the left-hand side of Eqs. (4) and 5 should be scaled by $\tau_{p} / \tau_{p^{*}}$ and thus our results would represent a lower limit on $\Delta E^{*}$ [it does not affect the outcome of the relative contributions of Rashba and Dresselhaus interactions]. In this case, the momentum scattering lifetime is determined from the measured mobility according to $\tau_{\mathrm{p}}=\mu m^{*} / e$ where $m^{*}(E, T)$ is the temperature and energy dependent effective mass from the Kane model incorporating the temperature dependence of $E_{F}$ and the fundamental band gap $E_{g}[10,24,25]$. The temperature dependence of $E_{g}$ used for calculating the band-edge effective mass, $m^{*}(0, T)=$ $m^{*}(0,0) E_{g}(T) / E_{g}(0)$ includes just the dilatational contribution[26, 27]:

$$
E_{g}(T) \leftarrow E_{\mathrm{g}}^{\text {dil }}(T)=E_{g}(0)+5.17 \times 10^{-7} T^{2}-9.25 \times 10^{-9} T^{3}+3.81 \times 10^{-11} T^{4}-5.12 \times 10^{-14} T^{5}
$$

The resulting temperature dependence of the momentum scattering lifetime $\tau_{\mathrm{p}}$ is given in Fig. 2(b). Sample A exhibits a temperature dependence characteristic of phonon scattering, while sample B shows the opposite dependence with overall lower values of $\tau_{\mathrm{p}}$ which we attribute to the increased screening of ionized impurities in the QW with increasing $\mathrm{n}$ [28]. The temperature dependence of the spin lifetimes are shown in Fig. 2(c). Fig. 2(d) shows the corresponding $\alpha^{*}$ calculated according to Eq. (5) using experimental values taken from Figs. 2(a-c). As expected from the symmetric doping scheme, for sample B $\alpha^{*}$ is somewhat lower.

Calculations of the Rashba and Dresselhaus coupling parameters $\alpha, \beta$, and $\gamma$ in similar InSb/InAlSb QWs have been performed[22] at low temperatures using an SP model for the bandprofile, and a k.p model following the approach of Pfeffer and Zawadzki[10]. These calculations, repeated here for a range of temperatures, contain no fitting parameters, but are simplified in the sense that they do not take into account non-parabolicity or second subband occupancy (which is small in these narrow QWs). As for the effective mass, the temperature dependence of band gap used in the calculations of $\alpha, \beta$, and $\gamma$ was the dilatation part only $E_{\mathrm{g}}{ }^{\mathrm{dil}}(T)$. Note also that the temperature dependence of $n$ predicted by the SP solver [shown as dashed lines in Fig. 2(a)] is slightly different from that measured experimentally. In figure 3 we show the calculated Rashba and Dresselhaus terms in the form seen on the right hand side of Eq. (5) for sample A and B. As expected for the case of a one-sided modulation doped QW, the Rashba term in sample A dominates over the whole temperature range, giving a strong indication that the approximation $\alpha^{*} \sim \alpha$ is valid in this case. This result is illustrated more clearly in Fig. 3 inset which shows the relative Rashba contributions $\alpha / \alpha$. Proceeding in this manner, we obtain an experimental Rashba parameter $\alpha \sim 0.06-0.09 \mathrm{eV} \AA$ in the temperature range 77-300 K. In contrast, for sample $\mathrm{B}$, we find $\alpha^{*}>>$ and the same treatment can not be applied. Nevertheless, experimental values of $\alpha^{*}$ for samples $\mathrm{A}$ and $\mathrm{B}$ are surprisingly similar when considering that the Rashba term for sample B should be much reduced. To understand this result we need to consider the nature of the Te doping in the two QWs. Recall that sample B is uniformly doped and therefore $\tau_{\mathrm{p}}=\tau_{1}=\tau_{3}$, whereas for a modulation doped sample $\mathrm{A}$ we have $\tau_{\mathrm{p}}=\tau_{1}>\tau_{3}$ (we find $\tau_{\mathrm{p}}=$ $\left.\tau_{1} \approx 5 \tau_{3}\right)[20,22]$. Therefore, in the case of sample $\mathrm{B}$ an increased component of the momentum scattering perpendicular to the QW would bring into play the additional Dresselhaus term on the right hand side of Eq. (5) increasing the value of $\alpha^{*}$. It should be noted that the ability of this technique to determine the Rashba parameter is dependent on the correct choice of $\tau_{3} / \tau_{1}$. The ratio we used for sample A was calculated for remote ionised impurity scattering, and the same value was used for all temperatures. It might be argued that phonon scattering should be important at high temperatures, 
Experimental determination of the Rashba Coefficient in InSb/InAlSb quantum wells at zero magnetic field and elevated temperatures

however, the almost identical measured values of $\alpha^{*}$ for the two very different wells suggests that our choice of $\tau_{3} / \tau_{1}$ is sensible.

To verify that the values of $\alpha$ deduced for sample A from this experimental technique are reasonable, we can directly compare these results to the calculations described above, as shown in Fig. 4. Excellent agreement is obtained validating our assumptions about the spin and momentum relaxation mechanisms, and the theoretical model used for evaluating $\alpha, \beta$, and $\gamma[22]$. Furthermore, the agreement supports our assertion that electron-electron scattering is negligible. Previous simple predictions for the non-degenerate regime (which include only the Dresselhaus contribution to ZFSS) might lead to the conclusion that other spin relaxation processes are important[18, 23]. Overall, we have demonstrated the validity of this experimental approach as a measurement of the Rashba parameter.

While the measurement of $\alpha$ has important implications for spintronics it does not provide a direct figure of merit for a particular material system. As discussed, what is of greater relevance to the application of spintronics is the ability to modulate the electron spin via an applied electric field, characterized by the Rashba coefficient $\alpha_{0}$ (not to be confused with the Rashba parameter, $\alpha$ ). A large $\alpha_{0}$ enables the manipulation of spin populations using a gate electrode which is essential for spin dependent ballistic transport devices[2-4, 29]. To a first approximation, we can express the Rashba spin splitting by the relationship $\Delta E_{R}=2 \alpha_{0} e F k$. An estimate of $\alpha_{0}$ may be found from k.p theory $[9,30]$

$$
\alpha_{0}=\frac{\hbar^{2}}{2 m^{*}} \frac{\Delta_{s}\left(2 E_{g}+\Delta_{s}\right)}{E_{g}\left(E_{g}+\Delta_{s}\right)\left(3 E_{g}+2 \Delta_{s}\right)}
$$

where $m^{*}$ and $E_{g}$ are as before, and $\Delta_{\mathrm{s}}$ is the spin-orbit splitting energy. The general trend of this estimation is shown in Fig. 5. InSb has the narrowest band gap and largest spin-orbit splitting energy of the III-V semiconductors, and thus represents the upper limit to the magnitude of $\alpha_{0}$. Although it has been argued that an electron confined to a QW is in a bound state, and as such the average electric field perpendicular to the growth direction must be zero, the approach of Pfeffer and Zawadzki[10] (used here) elucidates the interface effects and shows that the spin splitting is not simply proportional to $F$ (for example, the term linear to $F$ contributes $\sim 16 \%$ to the total $\alpha$ for Sample A). However, we note that these interface terms are indirectly influenced by $F$ due to the resulting charge redistribution, and as we show below there is reasonable agreement between both applications of the k.p models[9-11]. Using $\alpha_{0}$ we are able to directly compare the merits of different materials systems as well are the technique employed in the measurement. In the case of the modulation doped sample A, we may estimate the built-in field experimentally by considering the electric field created by the separation of sheet charge of remote ionized donors and the electrons in the QW[31]:

$$
\langle F\rangle_{\exp } \approx e n / 2 \varepsilon_{r} \varepsilon_{0}
$$

where $\varepsilon_{r}$ is the relative dielectric constant of the InAlSb which we approximate as the value for InSb of 16.7[32]. The approximation of Eq. (8) is reasonable when compared to the theoretical $\langle F\rangle$ from the SP solver. This is shown in the inset of Fig. 1 where the dashed line corresponds to a theoretical $\varepsilon_{,} \varepsilon_{0}\langle F\rangle / n$ value of 0.5 obtained from the SP solver. The solid line refers to the decreasing value from 0.43 to 0.35 in the temperature range $77-300 \mathrm{~K}$, obtained by the approximation of Eq. (8). 
Experimental determination of the Rashba Coefficient in InSb/InAlSb quantum wells at zero magnetic field and elevated temperatures

\begin{tabular}{|c|c|c|c|c|c|c|c|}
\hline \# & Material & ref & exp. or theory & $\mathrm{T}(\mathrm{K})$ & $\alpha(\mathrm{eVA})$ & $F\left(10^{25} \mathrm{Vm}^{-1}\right)$ & $\alpha_{0}\left(\AA^{2}\right)$ \\
\hline 1 & \multirow{5}{*}{$\mathrm{InSb} / \mathrm{InAlSb}$} & $*$ & $\exp$ & $77-300$ & $0.06-0.09$ & $1.2-1.7$ & $316-332$ \\
\hline 2 & & [11] & $\exp$ & 2 & 0.14 & 1.1 & 783 \\
\hline 3 & & [12] & $\exp$ & 4.2 & 0.14 & $0.37-0.78$ & $1122-2364$ \\
\hline 4 & & {$[10,11]^{*}$} & theory & $77-300$ & $0.05-0.075$ & - & $370-439$ \\
\hline 5 & & {$[9,30]^{*}$} & theory ${ }^{\S}$ & $0,77-300$ & - & - & $513,304-310$ \\
\hline 6 & \multirow{2}{*}{ InAs/InAlAs } & [33] & $\exp$ & 0.28 & 0.22 & 6.25 & 220 \\
\hline 7 & & {$[9,30]$} & theory ${ }^{\S}$ & 0 & - & - & 103 \\
\hline 8 & \multirow{2}{*}{ GaAs/AlGaAs } & [8] & $\exp$ & $77-230$ & - & - & $7-15$ \\
\hline 9 & & {$[9,30]$} & theory ${ }^{\S}$ & 0 & - & - & 4.5 \\
\hline
\end{tabular}

Table 1 Summary of both theoretical and experimental (exp.) measurements of the Rashba coefficient for III-V semiconductor QWs. $\S$ Theoretical values independent of QW structure and calculated using accepted band parameters[34]. * The subject of this current work.

Measurements of the Rashba interaction in III-V materials, using both experimental and theoretical methods, are summarized in Table 1. Measurements \#1 \& \#5 are the experimental and theoretical values that are the focus of this work. Other experimental measurements of InSb/InAlSb QWs using Shubnikov de Haas $(\mathrm{SdH})$ oscillations (\#2) and electron spin resonance (\#3) have reported a much stronger Rashba interaction. Notably, while both works report similar $\alpha$, we estimate very different $\alpha_{0}$ when applying the simple parallel plate model, (Eq. 8). This originates from the lower sheet density $n$ in measurement \#3, and therefore without the use of calculations that include realistic confinement potentials, it is not possible to discount the contribution of the Dresselhaus terms to the ZFSS. As noted in Ref. [11], in the case of measurement \#2 the Zeeman contributions (which can be large in $\mathrm{InSb}$ ) were neglected in the analysis and it was suggested that these figures should only be used as an estimate. For completeness, experimental and theoretical values are also given for InAs and GaAs QWs. The SdH InAs experimental measurement \#6 was two times larger than that predicted for bulk InAs using Eq. 7 (\#7). As was the case for the all optical GaAs measurement \#8 taken in the temperature range $77-230 \mathrm{~K}$, though we have not included the predicted values at these temperatures caused by changes in $E_{\mathrm{g}}$.

\section{CONCLUSION}

By measuring the spin dynamics of degenerately doped InSb/InAlSb QWs in zero applied magnetic field we have deduced the spin-orbit splitting $2 \alpha^{*} k_{F}$ at elevated temperatures without the influence of the large Zeeman Effect. In the case of asymmetrically doped samples, we were able to directly extract the Rashba parameter $\alpha$. A model was developed with no adjustable parameters for the spin dynamics when the Rashba contribution dominates, and our measurements show very good agreement with it where it applies. For an asymmetric InSb/InAlSb QW at room temperature dominated by the Rashba interaction, we have extracted Rashba parameter and coefficient of $\alpha=0.09 \pm 0.1 \mathrm{eVA}$ and $\alpha_{0}$ $=350 \pm 50 \AA^{2}$, respectively which are in good agreement with theoretical expectations. Whilst this experimental measurement is lower than previously reported for InSb QWs using techniques in nonzero magnetic fields, it remains larger than that reported for the other III-V compound semiconductors, consistent with long standing theoretical predictions. The results have clear device implications because regardless of the value of $\alpha$ the large Rashba coefficient $\alpha_{0}$ in InSb/InAlSb QWs, 
Experimental determination of the Rashba Coefficient in InSb/InAlSb quantum wells at zero magnetic field and elevated temperatures

implies that significant modulation of the spin splitting is possible with small changes of an applied electric field. Increased confidence in the value of this coefficient is critical to assess the spintronic potential of any particular material system and whether proposed spintronic concepts are realizable.

This work was supported by UK-EPSRC (SPRINGS - EP/C511999, EP/E055583 and EP/F021836). 
Experimental determination of the Rashba Coefficient in InSb/InAlSb quantum wells at zero magnetic field and elevated temperatures

FIG. 1 Calculated conduction band profiles. (a) Conduction band profile $\left(\mathrm{E}_{\mathrm{c}}\right)$ of the modulation doped QW (sample A) showing the Te $\delta$-doping layer 20nm above the well and (b) $E_{\mathrm{c}}$ profile of the uniformly doped QW (sample B). Also shown are the wavefunctions $(\psi)$ for the first QW sub-band. Inset: Calculated electric field in the modulation doped sample A from both the full SP solver (dashed line) and Eq. (8) (solid line).

FIG. 2 Experimentally measured parameters used to calculate the effective Rashba parameter as a function of temperature for samples A (solid squares) and B (solid circles). (a) sheet carrier concentration, $n$; (b) momentum scattering time $\tau_{\mathrm{p}}$ derived from electron mobility; (c) spin lifetime $\tau_{\mathrm{s}}^{[001]}$ and (d) the magnitude of the effective inversion asymmetry spin splitting parameter $\alpha^{*}$ determined from Eq. (6) using the experimental data of (a)-(c). Also shown on (a) are the predictions (dashed line) from the SP solver (see text for details).

FIG. 3 Contributions to the effective spin splitting parameter for samples A (squares) and B (circles). Calculated Rashba (solid symbols) and Dresselhaus contributions (open symbols) including both $\beta$ and $\gamma$ terms to the square of the effective inversion asymmetry spin splitting parameter, $\alpha^{*^{2}}$, as defined by Eq. (5) and assuming $\tau_{\mathrm{p}}=\tau_{1}=5 \tau_{3}$ appropriate for a modulation doped sample[20, 22]. Inset: The fractional contribution of the Rashba interaction $\alpha$ to $\alpha^{*}$ for both of the samples. As expected for Sample A the Rashba term is dominant for all temperatures.

FIG. 4 Comparison of $\alpha^{*}$ and $\alpha$ using experimental (solid squares) and theoretical (open symbols) techniques for Sample A.

FIG. 5 Estimated values of the Rashba coefficient as a function of energy gap $\left(E_{\mathrm{g}}\right)$ and spinorbit splitting energy $\left(\Delta_{\mathrm{so}}\right)$ using k.p model[9, 30] for a triangular QW, see Eq. (1). The position on this surface for III-V binary compounds are shown using the recommended parameters taken from Vurgaftman et.al. [34] and references therein $(\mathrm{T}=0 \mathrm{~K})$. 
Experimental determination of the Rashba Coefficient in InSb/InAlSb quantum wells at zero magnetic field and elevated temperatures

\section{REFERENCES}

1. Stern, N.P., et al., Current-induced polarization and the spin Hall effect at room temperature. Phys Rev Lett, 2006. 97(12): p. 126603.

2. $\quad$ Chen, H., et al., Spin-polarized reflection in a two-dimensional electron system. Applied Physics Letters, 2005. 86(3): p. 032113.

3. Khodas, M., A. Shekhter, and A.M. Finkel'stein, Spin polarization of electrons by nonmagnetic heterostructures: The basics of spin optics. Physical Review Letters, 2004. 92(8): p. 086602.

4. $\quad$ Rokhinson, L.P., et al., Spin separation in cyclotron motion. Physical Review Letters, 2004. 93(14): p. 146601.

5. Bychkov, Y.A. and E.I. Rashba, Properties of a $2 d$ Electron-Gas with Lifted Spectral Degeneracy. JETP Letters, 1984. 39(2): p. 78-81.

6. Ohkawa, F.J. and Y. Uemura, Quantized Surface States of a Narrow-Gap Semiconductor. Journal of the Physical Society of Japan, 1974. 37(5): p. 1325-1333.

7. Vasko, F.T., Spin Splitting in the Spectrum of Two-Dimensional Electrons Due to the Surface-Potential. JETP Letters, 1979. 30(9): p. 541-544.

8. $\quad$ Eldridge, P.S., et al., All-optical measurement of Rashba coefficient in quantum wells. Physical Review B, 2008. 77(12): p. 125344.

9. Silva, E.A.D.E., G.C. Larocca, and F. Bassani, Spin-Split Subbands and Magneto-Oscillations in Iii-V Asymmetric Heterostructures. Physical Review B, 1994. 50(12): p. 8523-8533.

10. Pfeffer, P. and W. Zawadzki, Spin splitting of conduction subbands in III-V heterostructures due to inversion asymmetry. Physical Review B, 1999. 59(8): p. R5312-R5315.

11. Gilbertson, A.M., et al., Zero-field spin splitting and spin-dependent broadening in highmobility InSb/In1-xAlxSb asymmetric quantum well heterostructures. Physical Review B, 2009. 79(23): p. 235333.

12. Khodaparast, G.A., et al., Spectroscopy of Rashba spin splitting in InSb quantum wells. Physical Review B, 2004. 70(15): p. 155322.

13. Dedigaina, A.R., et al. Measurement of the Dresselhaus and Rashba spin-orbit coupling via weak anti-localization in InSb quantum wells. in 13th International Conference on Narrow Gap Semiconductors. 2007. Guildford, ENGLAND: Springer.

14. Zawadzki, W. and P. Pfeffer, Spin splitting of subband energies due to inversion asymmetry in semiconductor heterostructures. Semiconductor Science and Technology, 2004. 19(1): p. R1-R17.

15. Dresselhaus, G., Spin-Orbit Coupling Effects in Zinc Blende Structures. Physical Review, 1955. 100(2): p. 580-586.

16. Leyland, W.J.H., et al., Enhanced spin-relaxation time due to electron-electron scattering in semiconductor quantum wells. Physical Review B, 2007. 75(16): p. 165309.

17. Averkiev, N.S., L.E. Golub, and M. Willander, Spin relaxation anisotropy in twodimensional semiconductor systems. Journal of Physics-Condensed Matter, 2002. 14(12): p. R271-R283.

18. Tackeuchi, A., et al., Electron spin-relaxation dynamics in GaAs/AlGaAs quantum wells and InGaAs/InP quantum wells. Japanese Journal of Applied Physics Part 1-Regular Papers Short Notes \& Review Papers, 1999. 38(8): p. 4680-4687.

19. Crooker, S.A. and D.L. Smith, Imaging spin flows in semiconductors subject to electric, magnetic, and strain fields. Physical Review Letters, 2005. 94(23): p. -.

20. Averkiev, N.S., et al., Spin-relaxation anisotropy in asymmetrical (001) AlxGa1-xAs quantum wells from Hanle-effect measurements: Relative strengths of Rashba and Dresselhaus spin-orbit coupling. Physical Review B, 2006. 74(3): p. 033305. 
Experimental determination of the Rashba Coefficient in InSb/InAlSb quantum wells at zero magnetic field and elevated temperatures

21. Kainz, J., U. Rossler, and R. Winkler, Anisotropic spin-splitting and spin-relaxation in asymmetric zinc blende semiconductor quantum structures. Physical Review B, 2003. 68(7): p. 075322.

22. Gilbertson, A.M., et al., Zero-field spin splitting and spin lifetime in $n$-InSb/In1-xAlxSb asymmetric quantum well heterostructures. Physical Review B, 2008. 77(16): p. 165335.

23. Litvinenko, K.L., et al., Spin relaxation in n-InSb/AlInSb quantum wells. New Journal of Physics, 2006. 8: p. 49.

24. Pfeffer, P. and W. Zawadzki, Conduction Electrons in Gaas - 5-Level K.P Theory and Polaron Effects. Physical Review B, 1990. 41(3): p. 1561-1576.

25. Zawadzki, W., Electron-Transport Phenomena in Small-Gap Semiconductors. Advances in Physics, 1974. 23(3): p. 435-522.

26. Litvinenko, K.L., et al., Temperature dependence of the electron Lande g factor in InSb and GaAs. Physical Review B, 2008. 77(3): p. 033204.

27. Zawadzki, W., et al., Temperature dependence of the electron spin $g$ factor in GaAs. Physical Review B, 2008. 78(24): p. 245203.

28. Pooley, 0.J., et al., Transport effects in remote-doped InSb/AlxIn1-xSb heterostructures. New Journal of Physics, 2010. 12: p. 053022.

29. Tarasenko, S.A., V.I. Perel', and I.N. Yassievich, In-plane electric current is induced by tunneling of spin-polarized carriers. Physical Review Letters, 2004. 93(5): p. 056601.

30. Pikus, F.G. and G.E. Pikus, Conduction-band spin splitting and negative magnetoresistance in A3B5 heterostructures. Physical Review B, 1995. 51(23): p. 16928.

31. Brosig, S., et al., Zero-field spin splitting in InAs-AlSb quantum wells revisited. Physical Review B, 1999. 60(20): p. R13989-R13992.

32. Wakaki, M., K. Kudo, and T. Shibuya, Physical Properties and Data of Optical Materials. 2007: CRC Press.

33. Heida, J.P., et al., Spin-orbit interaction in a two-dimensional electron gas in a InAs/AISb quantum well with gate-controlled electron density. Physical Review B, 1998. 57(19): p. 11911-11914.

34. Vurgaftman, I., J.R. Meyer, and L.R. Ram-Mohan, Band parameters for III-V compound semiconductors and their alloys. Journal of Applied Physics, 2001. 89(11): p. 5815-5875. 
Experimental determination of the Rashba Coefficient in InSb/InAlSb quantum wells at zero magnetic field and elevated temperatures

FIG. 1
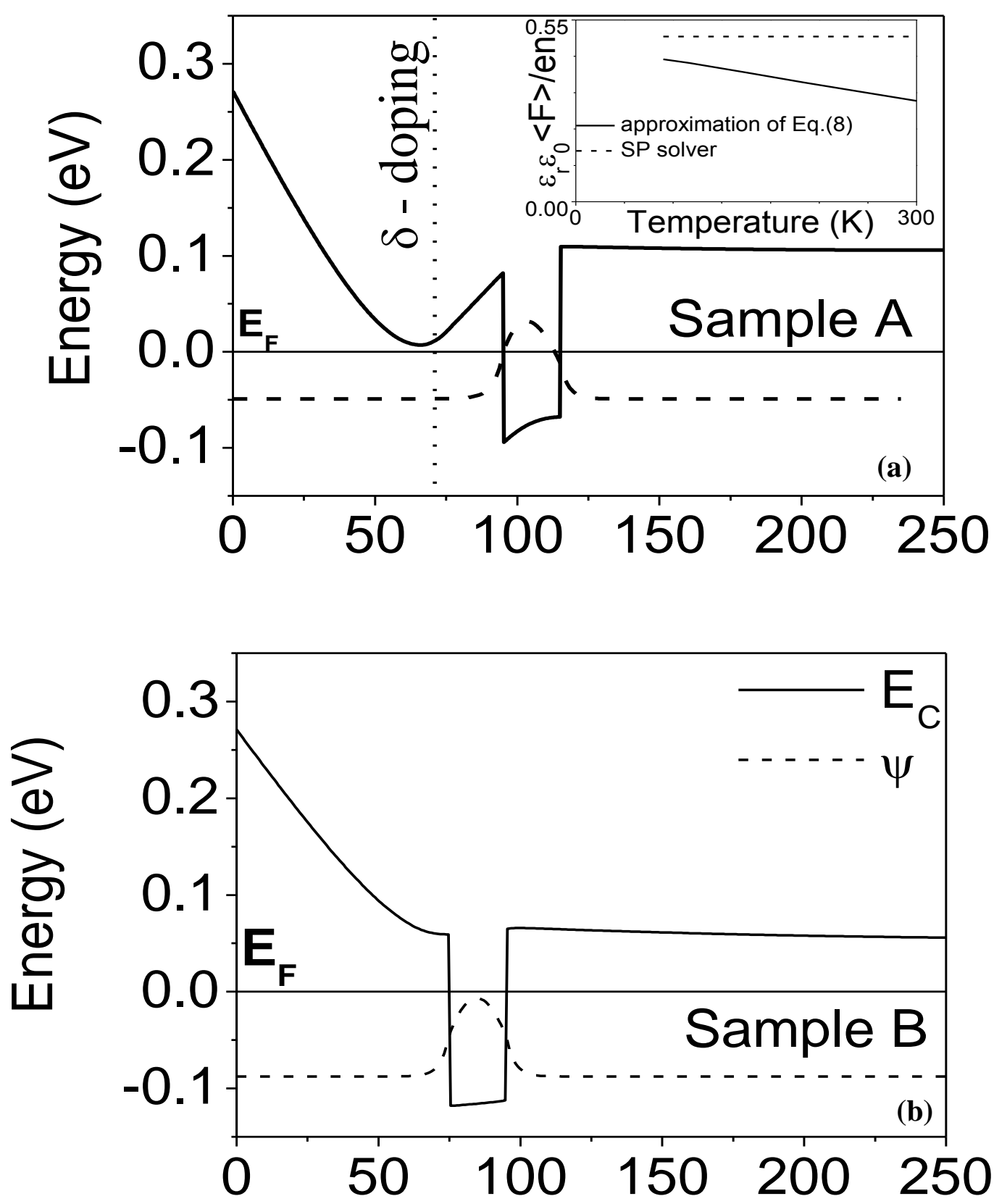

Distance from surface $(\mathrm{nm})$ 
Experimental determination of the Rashba Coefficient in InSb/InAlSb quantum wells at zero magnetic field and elevated temperatures

FIG. 2
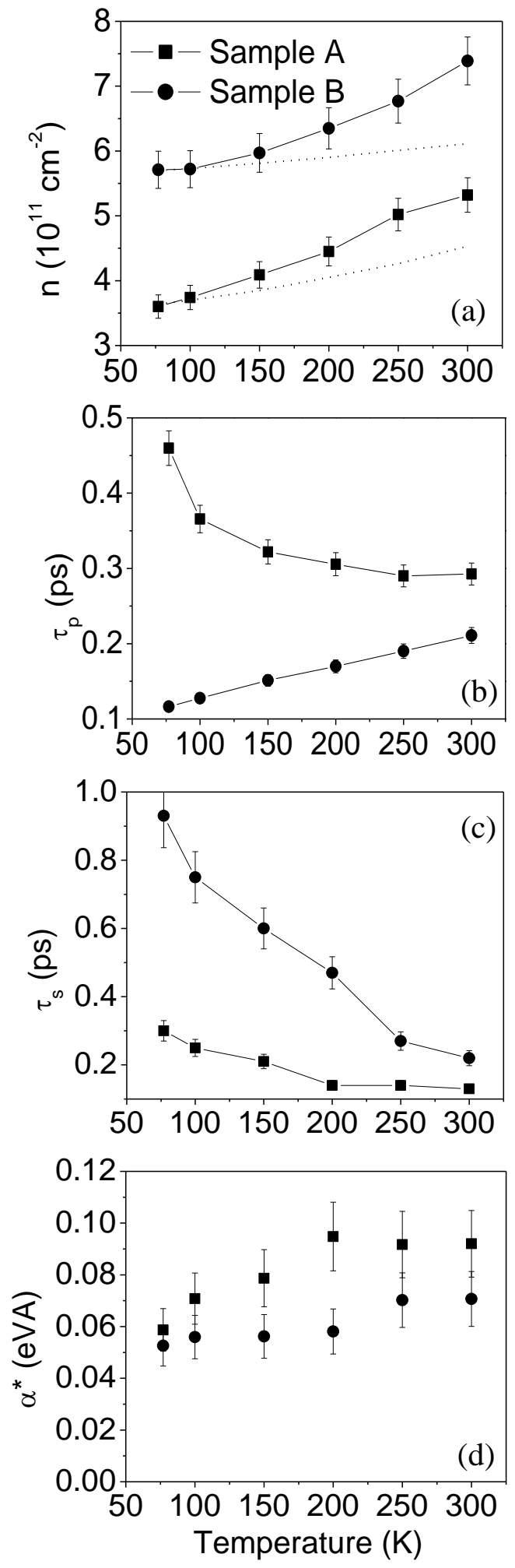


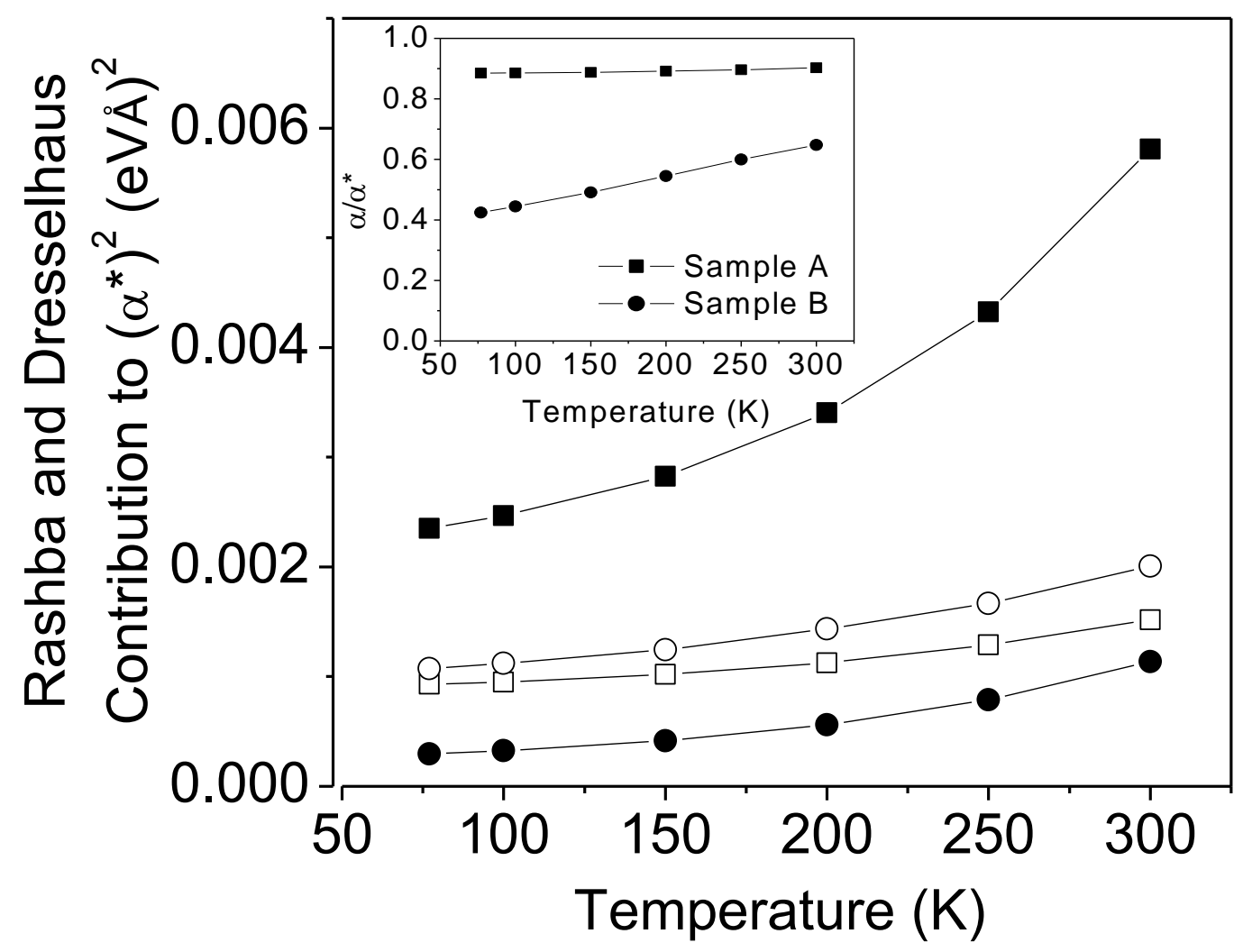

FIG. 3 
Experimental determination of the Rashba Coefficient in InSb/InAlSb quantum wells at zero magnetic field and elevated temperatures

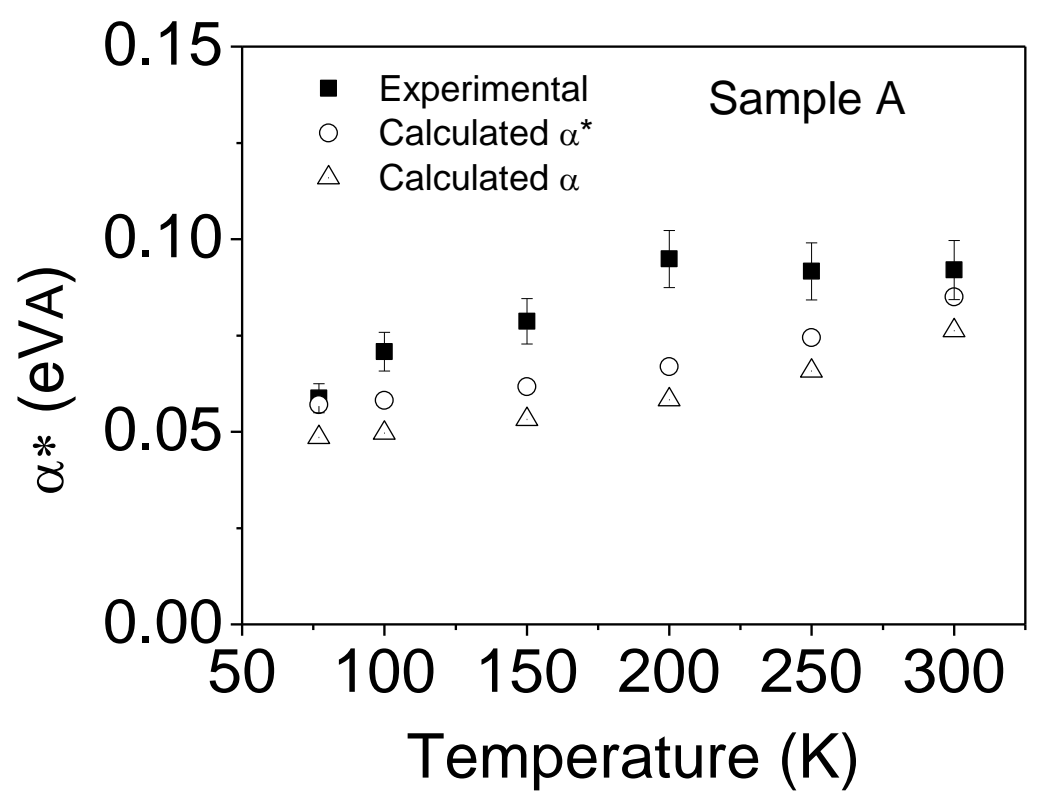

FIG. 4 
Experimental determination of the Rashba Coefficient in InSb/InAlSb quantum wells at zero magnetic field and elevated temperatures

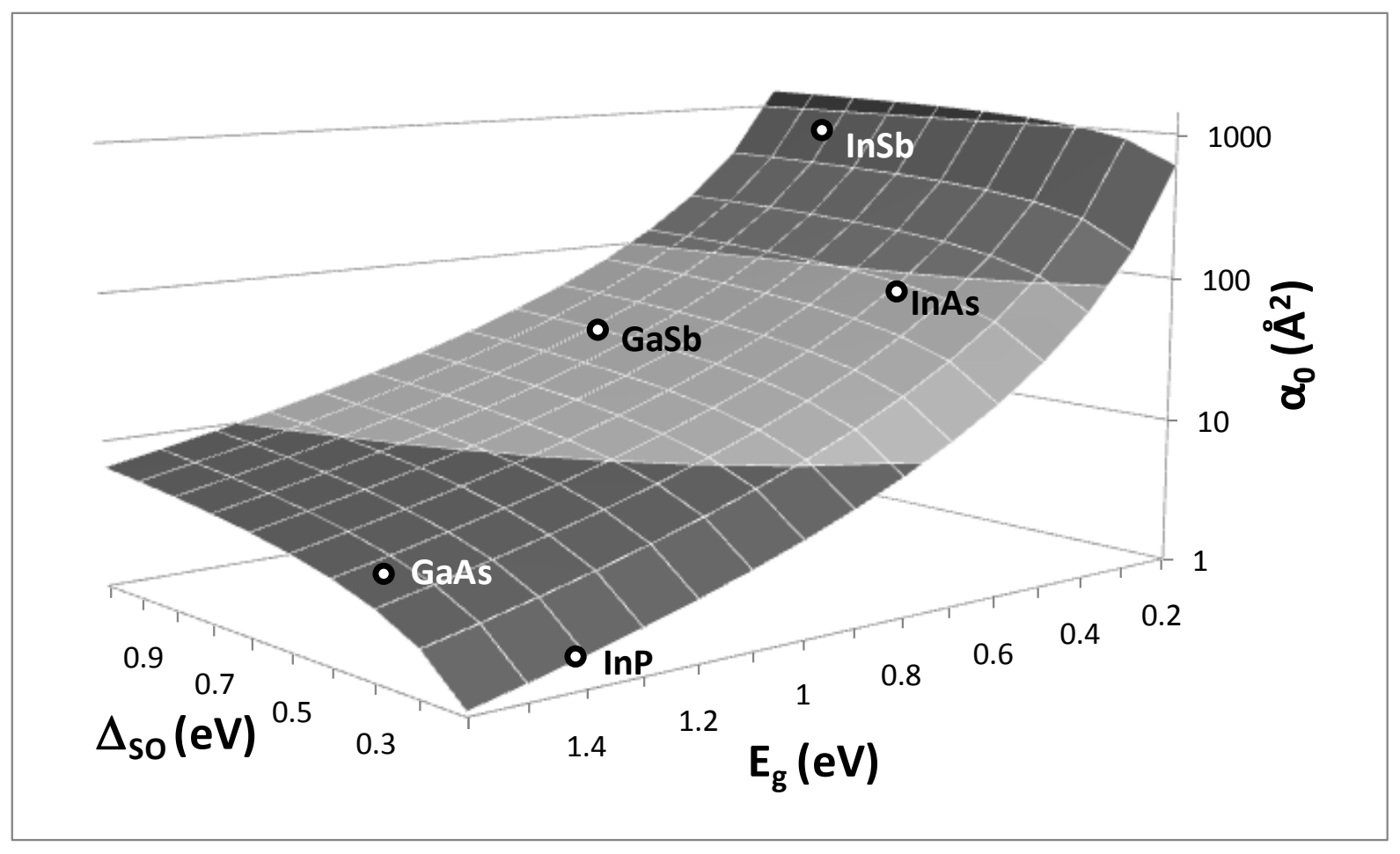

FIG. 5 